\title{
UNIDIRECTIONAL ROTATION NEURONES IN THE OP'TOMOTOR SYSTEM OF THE CRAB, CARCINUS
}

\author{
By C. A. G. WIERSMA* AND L. FIORE† \\ Department of Physiology, Medical Faculty, Rotterdam
}

(Received 5 October I970) $^{2}$

\section{INTRODUCTION}

As described in the previous paper (Wiersma \& Fiore, I97I), it is well known that position-sensitive elements in the statocyst organs exert a strong influence on the optomotor system of the stalked-eye decapod crustaceans. These influences can be very long-lasting in the small position-determining motor neurones. However, in the medium-sized more phasic motor fibres to each muscle more habituation occurs, and a certain degree of dir ectionality becomes evident (Wiersma \& Oberjat, I968.) The explanation of the latter phenomenon is not clear. It might be due to a preferred stimulation of the same sensory elements responsible for the maintained discharges, which are presumably the hair fibres whose tips are imbedded in the statolith. Alternatively, other sensory input with directional sensitivity may add to the discharge rate of the motor fibres. The evidence for the latter possibility comes from observations of Cohen (1955, 1960) in the lobster, Homarus. He found free-floating hairs in the statocyst chamber which he calls thread hairs and which respond to acceleration. In the crayfish and rock lobster we have failed to find motor fibres which could be exclusively stimulated by such a system (Wiersma \& Oberjat, r968; C. A. G. Wiersma, unpublished). In the crab, however, the discharges shown by the large motor fibres, described in the following pages, may well be triggered by this type of input.

\section{METHODS}

The preparations were as described in the previous paper. The fibres involved were large, but easily overlooked since they were normally quiescent. However, during strong excited states, their spikes appeared, and though always very limited in number they indicated the presence of a motor fibre with special properties. In addition, these fibres were often located in proximity to position fibres. Once this was ascertained, they were found in many instances.

For their investigation quick rotations were necessary. For testing purposes these were provided by holding the preparation on its base plate and turning it in various directions. For more precise studies the plate was mounted in various positions in the centre of a rotating wheel, which made possible a better control of rotations and stops. But this method has still several drawbacks, especially when unnatural starting positions are required for activation of the fibre.

In a number of experiments one (or both) statocysts was removed leaving the needle

- Present address: Division of Biology, California Institute of Technology, Pasadena, California 9i Iog, U.S.A.

$\dagger$ Present address: Istituto di Biologia Generale, Università di Pisa, Italy. 
in its place. This was accomplished by thrusting a blunt probe into the proximal antennule segment, breaking it off and then sealing the wound with bone wax. It was possible to check whether this procedure had interfered with the recording conditions by initiating a strong excited state and noting the presence or absence of the original impulses.

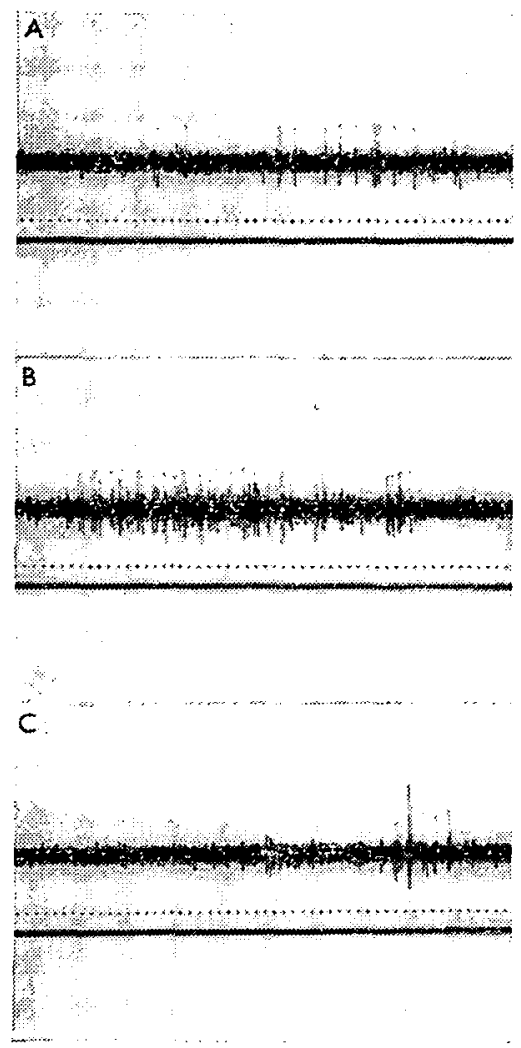

Fig. I. Anticlockwise unidirectional fast motor fibre. A, Discharges to a rotation of the animal in the dark at about $15 \%$. B, Same preparation at a speed of $90 \%$. C, Same, with rotation in the clockwise direction at $90 \%$, showing a discharge on the sudden stop. Time: $1 / 50 \mathrm{~s}$.

\section{RESULTS}

The fibres reacting only during fast turning movements were all of relatively large size, as shown by their action potentials. The first set found and most frequently studied consisted of two fibres which fired when the animal was rotated around its dorso-ventral axis. This is the same plane in which, when visual cues are present, movement excites the tonic optokinetic fibres. The large fibres fire equally well, however, in the dark as in light and do not respond when a striped drum is turned around the animal, no matter at what speed of rotation. They could be tested with the apparatus at hand more easily than other fibres of this class.

Their threshold for rotation of the animal was found to be much higher (in degrees/s) than for the optokinetic fibres, as no responses were obtained unless speeds of more than $10 \%$ were imposed. The fibres were unidirectional in that one responded to 
clockwise, the other to anticlockwise, rotation. After a rotation in the null direction a short-lasting discharge follows when the rotation is suddenly stopped. Firing always stopped during continued motion, but lasted longer with higher speeds. Fig. I shows discharges obtained with this method during rotation in the preferred direction and on stopping in the null direction.

When the body is placed in a different plane, e.g. vertically by putting the dorsoventral axis in a horizontal direction, and is rotated around this axis, the results are not different from those with the animal in the horizontal plane. Total removal of both antennules always led to a greatly diminished reactivity. In the great majority of the cases this was not due to a loss of the lead, since it was almost always possible to obtain a few impulses from what was clearly the same fibre by bringing the animal into a state of great excitement.

After the discovery of these phasic fibres on quick turns around the dorso-ventral axis we searched for similar fibres in other directions of rotation. Such fibres were indeed found and showed similar properties. Because of their short-lasting discharges, however, they are difficult to study. Furthermore, sudden stoppage of rotation in one direction will cause a discharge in the fibres which respond preferably to the other. Additionally, these fibres are less restricted with respect to their preferred direction than are the tonic fibres. This means that under certain circumstances of abnormal body positions the two fibres effecting antagonistic torques may be simultaneously firing, though one more strongly than the other. Their total number therefore remains somewhat of a problem, but it seems likely that there are in addition to the two mentioned, a head-up, a head-down, an eye-up-head-up, an eye-down-head-down, and an eye-up fibre. For the head-up rotation fibres it was found that, when the animal was mounted on its side, rotation in the direction towards head-up (in the normal position) caused about equal firing over the whole arc. This indicates that the failure of the fibre to fire in head-down positions is caused by a gravitational inhibitory effect. The fact that more impulses resulted when the eye from which the lead was taken was pointing upward, than when downward, is probably also due to gravity.

From their reactions alone the different fibres are difficult to distinguish from one another. For instance, the fibie for head-up rotation and the fibre for head-up-eye-up rotation, both showed a greater discharge rate when the animal was turned in the headup part of the arc than in the head-down part, though firing was still present in the latter, in contrast to the more phasic of the 'position' fibres. Therefore impulses present during rotation in the long axis are no certain indication that the experimental fibre is the head-up-eye-up one and not the head-up one. The fact that all fast unidirectional fibres can also be triggered by the excited state further complicates the picture, as does the discharge on sudden stops in the null direction. The main reason for accepting the presence of the two fibies is that they were often variously accompanied by either pure head-up or by head-up-eye-up position fibres. Under certain conditions a further complication arises in distinguishing them from the more phasic of the position fibres. Usually the latter show an hysteresis; that is, they fire much more frequently when the direction of the turn is from the rest position than from the upside-down position. In animals in which these medium-sized fibres reveal little or no tonic discharge during maintained positions, they behave in most respects like the unidirectional fibres, though the latter react only on faster speeds. 


\section{Effects of visual input}

In a number of experiments the effects of rotation were compared under normal room illumination and in darkness for the different classes of fibre. In all the phasic fibres, whether unidirectional or position-sensitive, no difference was found. Furthermore, light stimuli had no effect in one or the other eye or on different regions of the eye. Turning stripes around the eye in various directions to its axis was also without effect, and this was even true for the small positional fibres of the eye-up, head-up, head-up-eye-up, head-down and head-down-eye-down types. In this respect there is
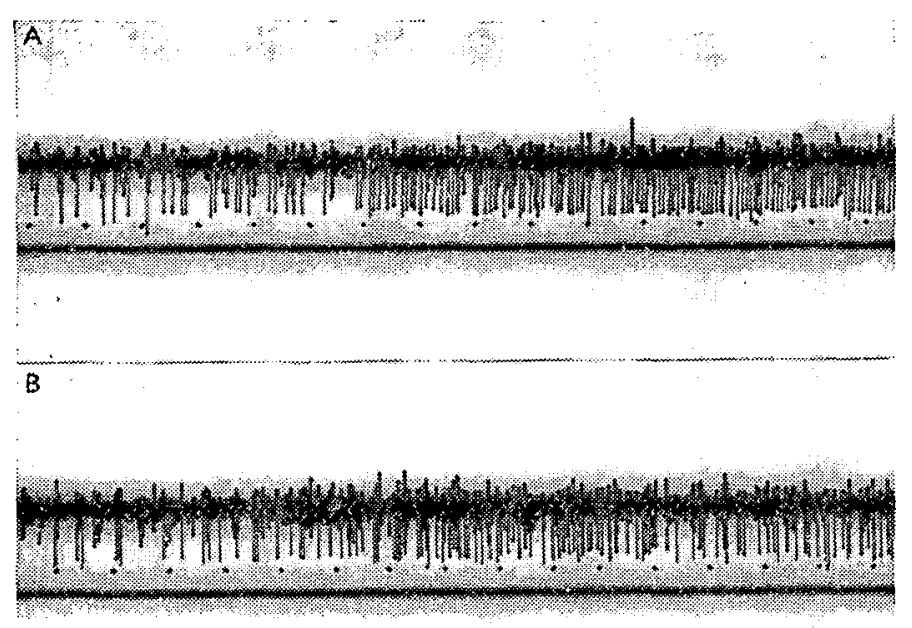

$\mathrm{C}$
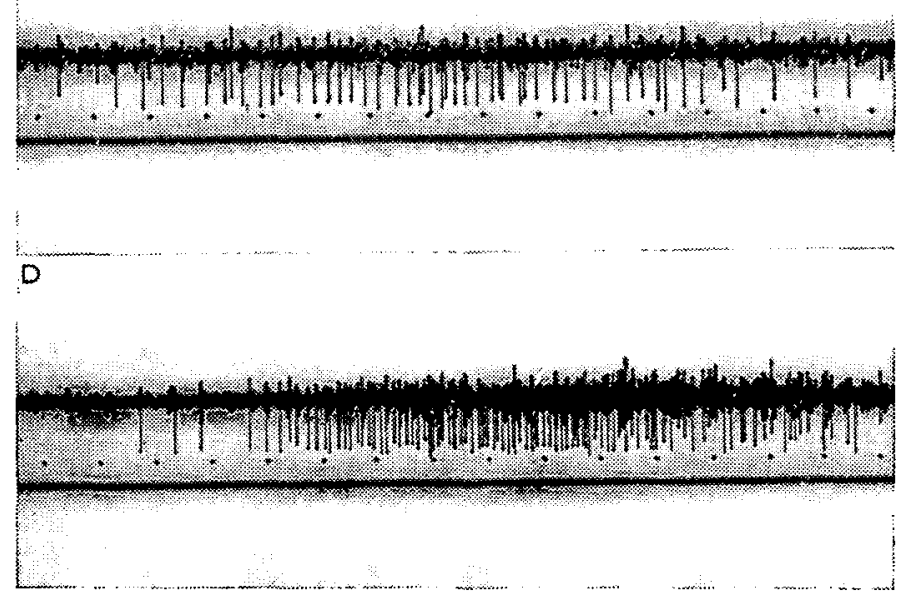

Fig. 2. Action potentials of a medium anticlockwise movement fibre responsive to both visual and mechanical stimulation. A, Response to turning a striped drum (with $\mathrm{I}$-in black and 3 -in white stripes) at a speed of $0 \cdot \mathrm{I}^{\circ} \mathrm{s}$. B, Same at a speed of $5 \% \mathrm{~s}$. C, Response to turning the animal at $5 \% \mathrm{~s}$ in the dark. D, Same at a speed of $90 \%$. Note that the discharge frequency is now as in $\mathrm{A}$. Time: $\frac{1}{10} \mathrm{~s}$. 
a difference between Carcinus on the one hand and the crayfish and lobster on the other the latter showing reactivity to light direction and to rotating stripes (Wiersma \& Oberjat, 1968; and unpublished).

In Carcinus there was only one set of fibres that reacted to both visual and statocyst input. These were medium-sized fibres reacting to rotations around the dorso-ventral axis. Because their existence was discovered rather late in the experimental series, the results are qualitative, and a further study of their interesting behavious should be rewarding. It was found that these fibres (both clockwise and anticlockwise were found) can be driven both by rotating a striped drum around the animal or by turning the animal in the dark in the opposite direction. For the latter the minimum speed necessary appears to be lower than that required to trigger the phasic unidirectional fibres. However, in order to obtain similar discharge frequencies the speed of rotation of the animal in the dark has to be considerably higher than that of the rotating drum in the light. What needs to be determined much more precisely is how much overlap there is in angular velocities under these two circumstances. The impression gained was that visual and rotational input sum when the animal is turned at an intermediate speed in the light before a standing striped background, since turning either the illuminated background or the animal in the dark at the same speed resulted in lower discharge frequencies. But an extensive study to obtain actual figures about this summation remains for the future. We obtained a single satisfactory record of one preparation, shown in part in Fig. 2. The observations on this were as follows. By turning the striped drum around the animal at a speed of $0.1 \% \mathrm{~s}$ a discharge frequency was gradually attained which led to eye flipbacks. Following this it took some ro s to restore maximal discharge rate. At $2 \%$ this required about $6 \mathrm{~s}$ after flipbacks, and at $5 \%$ about $3 \mathrm{~s}$. When faster speeds were tested the basic frequency remained low after an initial increase very shortly after onset, and no flipbacks occurred. When then tested with $3 \%$ the flipbacks were again present and the time to regain peak frequency was about $5 \mathrm{~s}$. Next the animal was turned by hand, in the drum, in light and darkness. At about $5 \%$ the frequency increased very rapidly in the light, whereas in darkness only a few impulses, at low frequency, were elicited. At higher speeds in darkness higher frequencies were obtained, and at about $90 \%$ the frequency was as high as at $5 \%$ on stripe turning alone. From these findings it is evident that low speeds of rotation in the dark will not elicit any impulses. At fast speeds, turning stripes are effective only at the very start, whereas on animal turning the influence of the mechanical input is more prolonged. It was estimated that on turning this animal at around $7 \%$ in the light before a striped background the two components would have shown maximal summation.

\section{DISCUSSION}

The finding of motor fibres which react to unidirectional movement without optical input, and the proof that these are at least primarily driven by the statocyst organs, open the possibility that their main input channel is provided by other sense organs than the 'normal' statocyst fibres. As stated, Cohen $(1955,1960)$ has shown that in the common lobster, Homarus, fluid motions in the statocyst chamber are perceived by special hairs, which float in the cavity and are longer than those glued to the statocyst. Such hairs may well be concerned with the input of the unidirectional fibres, since they 
would be equivalent to the ampullar hairs in the canals of the vertebrate vestibular system responsive to accelerations but not to position. They would also be expected to show a discharge in response to the deceleration of a rotation in the direction opposite to their preferred direction. This would lead to the observed discharge on sudden stopping of rotations in the null directions of the motor fibres. Again, in contrast to the precision found for the relationship between body (thus statocyst) position and the firing in the tonic fibres to the eye muscles, one would expect inaccuracy for these phasic fibres since fluid movements of a rotatory character might result from several factors other than those connected with the main rotatory direction, especially when such rotations were made in hand-held preparations, as was mostly the case. On the evidence available, however, the possibility cannot be excluded that during acceleration the fibres connected with the statocyst provide part or all of this input.

In the crayfish and rock lobster no output fibres of this type have been found, though it is likely that in both cases similar free hair fibres are present in the statocyst cavity. In the crayfish these have been found by Dr M. Hisada (personal communication). That these animals lack the fast unidirectional motor fibres may be explained by the fact that they do not rotate their bodies at anywhere near the speed that the crabs do. Their only quick motions are during escape swimming when the eyes in the crayfish are held forward, and the withdrawal muscle of the rock lobster appears also to contract. Presumably the same is true for Homarus, which raises the question as to whether the activity of the floating hairs is expressed in any output in these species. Possible outputs would be the faster of the two tonic nerve fibres innervating the different eye muscles, such as head-up and eye-up ones. If the floating hairs enhanced the output in these more phasic position fibres during rotation in their preferred direction and inhibit it when turned in the opposite one, the observed asymmetry would be explained.

The differences between the eye-muscle motor systems of various decapod species are an interesting example of how, by various means, the overall similar input-output relationships are changed to adapt to the specific needs of the animals. The addition of an extrafast motor fibre in crabs to the same musculature involved in other reflexes mirrors differences in motor innervation of various muscles of the appendages (see Atwood, I967). Of all species investigated, crabs have been found to show the most complicated relationships in this respect.

Such subsystems can also show specializations in their input channels. In Carcinus this aspect is well illustrated by the presence of the medium-sized optokinetic movement fibres which are sensitive to both visual and body-rotatory influences in the horizontal plane. When the optic input becomes ineffective due to 'blurring' during fast turns, the mechanical rotatory input will dominate and keep the eyes in the same position. The purely mechanically elicited discharge in the fastest fibres is probably a similar but much less precise mechanism. Another aspect of this is that the crab appears to lack visual input for the position fibres, whereas in the crayfish and rock lobster the eye-up and eye-down as well as the head-up and head-down sets of fibres are sensitive to both light intensity and stripe movement. This may be connected with the great changes of position of the eyecups in these crabs as compared with other species, which might make the presence of light reactions in more than one system confusing. 
The present findings and those in the previous paper (Wiersma \& Fiore, 1971) have stressed the considerable differences which exist between the optomotor systems of various decapod Crustacea, and have indicated that these differences have a functional basis. Comparative studies along these lines may further elucidate the phylogenetic relationship in one subsystem with specifiable though varying function.

\section{SUMMARY}

I. Among the optomotor fibres to the eye muscles in Carcinus a class was found which responds to unidiı ectional fast rotations around various body axes. All had large signals and are therefore of large diameter.

2. In one set of these fibres which fires especially for rotations around the dorsoventral axis, it could be shown that discharges take place especially during accelerations and that, when a rotation in the null direction is suddenly stopped, a short discharge occurs. The fibres for other axes behave in a similar manner.

3. For rotations around the ventro-dorsal axis, but not for other directions, mediumsized fibres are present which, in contrast to the fast fibres, respond to visual stimulation, as well as to body rotations in darkness, thus combining the input properties of the unidirectional fast rotatory and the unidirectional purely optokinetic small fibres. Their sensitivity to visual input is for low rotation velocities, to body rotations is for high rotation velocities.

The authors wish to express their appreciation to Professor Dr M. W. van Hof. These experiments were made possible by support from the Medical Faculty, Rotterdam, by grant GB 693 I X from the National Science Foundation to C. A. G. Wiersma, and by funds from the home Institutions.

\section{REFERENCES}

Atwood, H. L. (1967). Crustacean neuromuscular mechanisms. Am. Zool. 7, 527-51.

CoHEN, M. J. (1955). The function of receptors in the statocyst of the lobster Homarus americanus. f. Physiol., Lond. 130, 9-34.

COHEN, M. J. (I960). The response patterns of single receptors in the crustacean statocyst. Proc. Roy. Soc. Lond. $B$ I52, 30-49.

Wiersma, C. A. G. \& FIORE, L. (1971). Factors regulating the discharge frequency in optomotor fibres of Carcinus maenas. Y. exp. Biol. 54, 497-505.

Wiersma, C. A. G. \& OBERJAT, T. (1968). The selective responsiveness of various crayfish oculomotor fibers to sensory stimuli. Comp. Biochem. Physiol. 26, I-ı6. 
